\title{
Formación de Brigadas Médicos-Rescatistas de Respuesta Rápida Ante Catástrofes y Desastres Internacionales
}

\author{
Juan José López Lerena, Álvaro Tondo, Danilo Ipharraguerre, Carlos Nicola, Leandro Palomeque, Enrique Martinez
}

\begin{abstract}
RESUMEN
En Uruguay, la formación médica emergencia prehospitalaria de desastres y catástrofes es irregular. Por otro lado, los bomberos, están capacitados para búsqueda y rescate pero no para los primeros auxilios. Se propuso formar brigadas de respuesta rápida frente a emergencias complejas, catástrofes y desastres integradas por médicos, paramédicos y bomberos con el objetivo de optimizar la respuesta frente a estas situaciones y en definitiva lograr el objetivo supremo de salvar cuantas más vidas sea posible.
\end{abstract}

Material y método: Se creó un cronograma de actividades valorando: objetivo esperado, recursos humanos y materiales necesarios para lograr el objetivo, capacitación del personal. Cada módulo debía superar el $80 \%$ del resultado esperado. En 2008 y 2009 capacitamos en Osaka, J APON a 5 médicos y 10 oficiales bomberos. ${ }^{1}$ Estos, a su vez, formaron a 25 oficiales de bomberos 40 bomberos, 7 médicos y 15 enfermeros. Cada Brigada estaba conformada por 2 médicos, 2 enfermeros, 2 oficiales y 5 bomberos.

Durante el año 2010 se realizaron los módulos teoricopracticos para instrucción y capacitación., culminando con curso - taller con la supervisión de expertos japoneses, dos médicos expertos en TRAUMA y DESASTRES y tres oficiales de bomberos expertos en desastres (Grupo HEAVY de INSARAG ). ${ }^{2}$ La evaluación fue excelente. En la región son las primeras brigadas conformadas con estas características. Se espera que con la aplicación de esta nueva metodología de trabajo se logre el objetivo planteado. A la fecha se muestran tres intervenciones de estas brigadas con tiempos de respuesta mejores y mejor calidad de atención primaria de victimas.

Palabras clave: R escatistas, Desastres, Catástrofes, Módulo.

\begin{abstract}
In Uruguay, prehospital emergency medical training of disasters and catastrophes is irregular. In addition, firefighters are trained for search and rescue but not for first aid. It is proposed to form a rapid response brigades to complex emergencies, catastrophes and disasters built by doctors, paramedics and firefighters in order to optimize the response to these challenges and ultimately achieve the ultimate goal of saving as many lives as possible.
\end{abstract}

Materials and methods: A schedule of activities was created, assessing: Objective, human and material resources needed to achieve the objective, training of staff. Each module had to exceed $80 \%$ of the expected result. In 2008 and 2009 we trained 5 doctors and 10 firemen in Osaka, J apan. ${ }^{1}$ These, in return, trained 25 firefighter officials, 40 firefighters, 7 doctors and 15 nurses. Each brigade consisted of 2 doctors, 2 nurses, 2 firefighters officers and 5 firefighters. During the 2010 the theoretical and practical modules for education and training were wrtitten. Culminating the training with a course-workshop under the supervision of J a panese experts, two medical experts in disaster and trauma and three firefighter officials, experts in disaster (Group Heavy INSARAG). ${ }^{2}$ The evaluation was excellent. In the region are the first brigade formed with these characteristics. It is expected that the implementation of this new methodology will achieve the objective. To date, these interventions show three brigades with better response times and improved quality care of primary victims.

Keywords: Rescuers, Disasters, Catastrophes, Modules.

How to cite this Article: Lerena J J L, Tondo Á, Ipharraguerre D, Nicola C, Palomeque L, Martinez E. Formación de Brigadas Médicos-Rescatistas de Respuesta Rápida Ante Catástrofes y Desastres Internacionales. Panam J Trauma Critical Care E merg Surg 2012;1(2):72-75.

\section{Source of support: Nil}

\section{Conflict of interest: None}

\section{INTRODUCCIÓN}

La medicina del trauma, emergencias y desastres ha presentado cambios evolutivos significativos en los siglos XX y X XI. Los episodios bélicos del siglo XX, las guerras: de Corea, de Vietnam, primera y segunda guerra mundial, desarrollaron nuevas modalidades de asistencia médica en esas situaciones a nivel de campo, intentando mejorar la sobrevida de los soldados en combate. Por otro lado a fines del siglo XX principios del XXI, el desarrollo económico industrial y humano se ha hecho cada vez más complejo y la concepción de una 'medicina del trauma', ha ido cambiando hacia una medicina de las emergencias complejas y los desastres. Estos han cobrado innumerables cantidades de vidas, tanto sean desastres naturales como provocados por el hombre en las últimas décadas y la respuesta ante estas situaciones no siempre fue la adecuada. La concepción de hospital central terciario de trauma y desastres debió ser complementada con equipos sanitarios de respuesta rápida, cada vez más calificados, que a nivel del área prehospitalaria puedan efectivamente estabilizar victimas, cuantas más sean posibles y en definitiva salvar vidas y llevar a estas víctimas con chances de sobrevivir hacia las puertas de hospitales. Es por esto que en diferentes países desarrollados desde hace tiempo se viene aplicando programas de educación no curriculares en esta temática. ${ }^{3}$ En el U ruguay como integrantes del Servicio M édico de la Dirección Nacional de Bomberos trabajando en conjunto con los bomberos rescatistas vimos hace varios años la necesidad de integrar nuestros esfuerzos para la respuesta frente a estos incidentes complejos y desastres de forma más eficiente y segura

Paper presented in Free Research Competition at the SPT 24th Annual Congress in Asuncion, Paraguay on November 2011. 
Formación de Brigadas Médicos-R escatistas de Respuesta Rápida Ante Catástrofes y Desastres Internacionales

tanto para el personal actuante, como para las víctimas. L os conceptos teóricos de' la hora de oro en el trauma pre hospitalario', de los ' 10 min de platino' en las emergencias de PCR y demás ya estaban incorporados pero no existían las técnicas y destrezas necesarias para un trabajo coordinado, en equipo, eficaz y rápido frente a grandes desastres.

\section{OBJETIVO}

Es así que se propuso a las autoridades nacionales la formación del personal médico y paramédico y del personal de rescate de bomberos en el área especifica de respuesta rápida frente a Desastres y Catástrofes $\mathrm{Nacionales} \mathrm{e}$ Internacionales, en Japón, por intermedio de la A gencia J aponesa de Cooperación Internacional (J ICA ), país que por su historia tiene gran experiencia en este tipo de situaciones.

\section{MATERIAL Y MÉTODO}

Se decidió conformar seis brigadas de respuesta rápi da frente a situaciones de emergencias y desastres integradas por once individuos cada una y personal de relevo. Estarían integradas por 2 médicos, 2 enfermeros, 2 oficiales de bomberos y 5 bomberos cada una,en total 11 integrantes. Dicho personal durante los años 2008 y 2009 fueron formados en la ciudad de OSA KA, Japón en el Hospital Sanseikay Senri, hospital terciario de referencia en trauma y desastres en la región y en el departamento de bomberos de O saka, Japón respectivamente. ${ }^{1}$

L a formación incluyó el siguiente programa deformación médica, M odalidad intensiva de 12 horas diarias durante 31 días en diferentes instituciones del lugar tanto en hospitales de tercer nivel como de segundo nivel y grupos especiales de búsqueda y rescate; Grupo D-M AT; G rupo Haz-M at, Grupo de Rescate Internacional, G rupo de Rescate de D efensa Civil.

Cursos de resucitación cardiovascular básica y avanzada, cursos de rescates en estructuras colapsadas y espacios confinados, curso de entrenamiento en manejo del trauma prehospitalario (este curso es de manejo médico-quirúrgico con atención médico-quirúrgica de emergencia en el área prehospitalaria en situaciones de desastres y pacientes atrapados).

El grupo de paramédicos participó de las mismas actividades y además del cuerpo de paramédicos de rescate de Japón (JPR), Institución líder a nivel mundial en esta área y los grupos de bomberos participaron en las actividades de entrenamiento en búsqueda y rescate en diferentes situaciones tanto en los grupos de defensa civil como en los de bomberos de $D$ pto de bomberos con personal capacitado y acreditado por INSA RA G para rescates internacionales. ${ }^{2}$ También se recibieron los cursos PRIM AP de OFDA para primera respuesta con materiales peligrosos y curso de primera respuesta en incidentes radiológicos y nucleares.

\section{PLANIFICACIÓN DE LA RESPUESTA MÉDICA FRENTE A LA EMERGENCIA}

Durante el año 2010 en U ruguay estas brigadas realizaron maniobras prácticas de trabajo de campo en diferentes regiones del país y actualizaciones periódicas de clases teóricas.

Finalizamos con curso intensivo en el mes de noviembre del 2010 donde se realizó un encuentro entre los brigadistas y los médicos expertos designados por JICA, Japón y tres bomberos con acreditación INSA RA G HEAVY designados por el gobierno central de J apón con el D pto de bomberos de Tokio. Estos concurrieron a U ruguay en donde se real izaron el siguiente cronograma de actividades que se detallan en el esquema. ${ }^{2}$

\section{ELEMENTOS GENERALES PARA LA CONFOR- MACIÓN DE UNA BRIGADA DE RESPUESTA RÁPIDA ANTE SITUACIONES DE DESASTRES ${ }^{3}$}

Rol del rescatista, rol del médico, rol del enfermero 0 paramédico Trabajo en equipo.

Soporte vital básico concepto y definición conceptos anatómicos y fisiológicos concepto fisiopatológico, cadena de supervivencia, masajes cardíacos, ventilación boca a boca, dispositivos de ventilación de barrera.

U so de fibrilador externo automático. Fundamentos: A plicaciones en adultos y niños, situaciones especiales, niños y embarazadas, cuando detenerse, estaciones de prácticas de RCP y del uso del DEA para todos los participantes con muñecos simuladores y simuladores de fribilación.

Se realizaron otros módulos soporte vital avanzado. Fundamentos, nociones anatómicas y fisiológicas de la via aérea; distintos tipos de cánulas de intubación, cánulas de mayo: máscara, etc.

Técnica de intubación orotraqueal, administración de oxígeno, pautas de administración parenteral de fluidos y drogas; técnica de punción venosa, técnica de punción ósea, punción crico-tiroidea. Vía aérea dificultosa; reposición de fluidos en pacientes con shock hipovolémico, manejo del monitor de fibrilador, arritmias cardíacas más frecuentes y uso de drogas antiarrítmicas: control de hemorragias, control de fracturas, uso del equipo antishoch, férulas inflables y chalecos de estricación.

\section{MÓDULO RESCATE EN ESTRUCTURAS COLAPSADAS}

Definición, diferencia entre rescate $(R E C)$ y búsqueda y rescate (BREC). Como identificar a los espacios vitales aislados. Como asegurar la escena y los posibles riesgos para la seguridad del rescatista y la zona caliente. 
Seguridad en la escena y equipo de rescate: primeros auxilios; seguridad de la víctima; control de la vía aérea; control de hemorragias; Fijación y extracción segura de víctimas; levantamientos de cargas; aplicación de medios tecnológicos (P-SAR) y tarea con perros K -SA R. ${ }^{4}$

\section{NORMAS INTERNACIONALES PARA EL TRABA- JO EN SITUACION DE DESASTRES CON MÚLTI- PLES VÍCTIMAS}

Empaquetamiento de víctimas y traslados a zonas seguras; búsqueda y localización de víctimas; maniobras prácticas; practicas de extracción de víctimas en situación de estructuras colapsadas mediante uso de diferentes materiales con que se cuenta.

\section{ESTACIONES DE RESCATE}

(1) búsqueda y localización; (2) empaquetamiento y traslado;

(3) ejercicios simulados.

\section{RESCATE EN ESPACIOS CONFINADOS DEFINICIÓN}

Como asegurar la escena y los posibles riesgos para la seguridad de los rescatitias y la zona caliente siguiendo un protocolo básico de actuación. Maniobras mecánicas de emergencia a realizar, control de via aerea, control de hemorragias: Práctica en diferentes situaciones de espacios confinados mediante la utilización de diferentes materiales con que se cuenta: Técnica de ingreso a espacios confinados, técnicas de acenso y descenso autónomo individual y asistida por medio de compañero, instalación y utilización de tripode y los accesorios para la tarea de rescate.

\section{CONCEPTOS GENERALES PARA LA CONFOR- MACION DE UNA BRIGADA}

SEGURIDAD del equipo de rescate, conocimientos y normas de seguridad internacionales desplazarse en una zona de desastres.

PANICO en países extranjeros en situación de múltiples víctimas.

A UTOGESTION de la B rigada de R escate en situaciones de Desastre en países extranjeros. Comunicaciones, Contacto con autoridades locales (Oficiales, Zonal es, J efes de tribus, etc.). U so de medios y personal local de zona de desastres (traductor, religioso, jefe local, herramientas locales).

CRITERIOS de permanencia y/o evacuación de la BRIGADA (alimentos, M edicinas, etc.). ${ }^{5}$

\section{EJERCICIOS SIMULADOS}

Demostración de trabajo de rescate real izado por los alumnos con evaluación de los expertos. Se realizó simulacro de accidente con múltiples víctimas en el A ereopuerto $\mathrm{N}$ acional de Carrasco con la participación de las seis brigadas y equipo de emergencia del aeropuerto y análisis por los expertos internacionales japoneses. Se realizaron disertaciones teóricas sobre Soporte Vital Avanzado, sobre situaciones de emergencias y rescates en A sia y J apón a cargo de los expertos japoneses en el marco del XIV CONGRESO de la SPT M ontevideo ,U ruguay N oviembre 2010. Se realizó eval uación teórico práctica de todos los módulos y de las destrezas adquiridas, tanto a nivel de Japón como de la conformación de las B rigadas de Respuesta Rápida en U ruguay.

\section{CONCLUSIONES}

Este proyecto que comenzó en el año 2008, se vio concretado en el año 2010 con la promoción de seis B rigadas de Respuesta Rápida ante Catástrofes y D esastres Internacionales integrada por médicos, paramédicos y rescatistas. Se destaca que estas B rigadas obtuvieron acreditación internacional de JICA -J apon y del Gobierno de J apón por medio de su embajador en U ruguay.

A ctualmente están en período de evaluación para presentar su candi datura a integrar los equipos de INSA RA G.

Este tipo de brigada es única en la región y en muchas partes del mundo y pretende ser una brigada de auto-gestión en respuesta a situaciones de desastre internacional donde no solo se necesita la tarea de búsqueda y rescate sino de atención médica e inmediata tales como los casos de los terremotos de Haití o de China.

La formación de estas brigadas a nivel nacional espera disminuir las tasas de morbilidad y mortalidad en accidentes con múltiples víctimas y desastres obteniendo tasas de sobrevida similares a los países que presentan estas brigadas.

Actualmente se han desarrollado dos maniobras en el período de verano del 2011 con participación de dos brigadas en incendios forestales en departamentos del este del país actuando las mismas con autonomía por 10 días cada una. L as mismas presentaron óptimo desarrollo con auto-cuidado y auto-gestión de sus integrantes y asistencia a víctimas de quemaduras y traumatismos leves en la zona sin necesidad de traslados a centros regionales optimizando así una respuesta frente a la emergencia.

\section{REFERENCIAS}

1. JICA U ruguay. Programa B ecas Formación 2008 y 2009

2. JICA U ruguay. Programa de A sistencia Técnica Expertos 2010. 
3. INSA RG. Guías y M etodologías de A cción 2010.

4. USAID-INSARAG. Reunión San José Costa Rica 2005. Criterios para Acreditación de Equipos BREC (USAR) para Latinoamérica.

5. OCHA. Safety and Security for National Humanitarian Workers.

\section{ACERCA DE LOS AUTORES}

\section{Juan Jose Lopez Lerena (Correspondencia)}

Departamento de Sanidad, Centro Rescate Sanitario Internacional de B omberos, M ontevideo; Dirección N acional de Sanidad Policial, M inisterio del Interior, M ontevideo, Uruguay, e-mail: Iopezlerena@ gmail.com

\section{Alvaro Tondo}

Departamento de Sanidad, Centro Rescate Sanitario Internacional de B omberos, M ontevideo; Dirección Nacional de Sanidad Policial, M inisterio del Interior, M ontevideo, U ruguay

\section{Danilo Ipharraguerre}

Departamento de Sanidad, Centro Rescate Sanitario Internacional de Bomberos, M ontevideo; Dirección Nacional de Sanidad Policial M inisterio del Interior, M ontevideo, U ruguay

\section{Carlos Nicola}

Comando de Oficiales de Rescate Internacional, Departamento Bomberos, M ontevideo; Dirección N acional de B omberos, M inisterio del Interior, M ontevideo, U ruguay

\section{Leandro Palomeque}

Comando de Oficiales de Rescate Internacional, Departamento Bomberos, M ontevideo; Dirección N acional de B omberos, M inisterio del Interior, M ontevideo, U ruguay

\section{Enrique Martinez}

Departamento de Sanidad, Centro Rescate Sanitario Internacional de Bomberos, M ontevideo; Dirección Nacional de Sanidad Policial M inisterio del Interior, M ontevideo, U ruguay 